\title{
DIAGNOSIS
}

\section{D-dimer assay was useful for ruling out suspected pulmonary embolism}

Ginsberg JS, Wells PS, Kearon C, et al. Sensitivity and specificity of a rapid whole-blood assay for $D$-dimer in the diagnosis of pulmonary embolism. Ann Intern Med. 1998 Dec 15; 129:1006-11.

\section{Questions}

How accurate is a D-dimer assay in patients with suspected pulmonary embolism (PE)?

\section{Design}

A blinded comparison of $\mathrm{D}$-dimer assay resilts, ventilation-perfusion $(\mathrm{V} / \mathrm{Q})$ lung scanning, and bilateral compression ultrasonography (CU) with watchful waiting ( $3 \mathrm{mo}$ ).

\section{Setting}

\section{Canadian tertary care hospitals.}

\section{Participants}

1177 consecutive adults (mean age 53.4 y, $59 \%$ women) who were referred for suspected PE. Exclusion criteria were suspected upper-extremity deep venous thrombosis, no symptoms within the previous 48 hours, receipt of anticoagulants for 72 houss, limited life expectancy, or contraindication to contrast media.

\section{Description of tests and diagnostic standard}

History and physical data were used to classify patients as having a high, mod-

\section{Commentary}

The diagnosis of acute PE remains troublesome for physicians because the signs and symptoms of this condition are not specific (i.e., many signs and symptoms that suggest PE occur in the absence of PE). Tests that allow confident exciusion of acute $\mathrm{PE}$ are very usefal. When a physician suspects acute $\mathrm{PE}$, normal V/Q scan allows anticoagulants to be withheld with a very low likelihood of symptomatic thromboembolism during 3 months of followmup (1). Unfortunately, more than half of patients with suspected PE have abnomal, but nondiagnostic, lung scans. Thus, a tese that allows a conficent decision to be made about withholding anticoagulation therapy when lung scans are not diagnostic would be enornously heipful. Negative serial $e x$ -

erate, or low pretest probability for $\mathrm{PE}$. $\mathrm{V} / \mathrm{Q}$ lung scans and bilateral CU from the common femoral vein to the calf trifurcation were done within 24 hours. Patients with nondiagnostic $V / Q$ scans or high-probability $\mathrm{V} / \mathrm{Q}$ scans and a low pretest probability had futher testing done using a complex algorithm. A D-dimer assay was done concurrently with initial testing. All patients were followed for 3 months.

\section{Main outcome measures}

Diagnostic test properties for $D$-dimer assay in patients with various combinations of pretest probabilities, $\mathrm{V} / \mathrm{Q}$ lung scans, and bilateral CU results.

\section{Main results}

$17 \%$ of patients had PE. For all patients, $\mathrm{D}$-dimer assay results had a sensitiviry

Test properties of $\mathrm{D}$-dimer assay for pulmonary embolism $(\mathrm{PE})^{*}$

Variable PE rate Sensitivity Specificity (CD +LR (CI) -LR (CD)
$(95 \% \mathrm{CI})$

Pretest probability

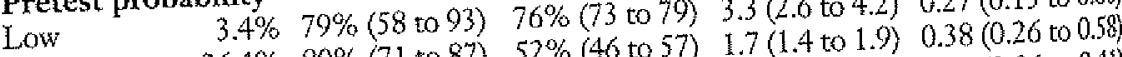
$\begin{array}{lllll}\text { Moderate } \quad 26.4 \% 80 \%(71 \text { to } 87) & 52 \%(46 \text { to } 57) & 1.7(1.4 \text { to } 1.0) & 0.38(0.26 \text { to } 0.41\end{array}$

Lung scan

$1.3 \% 75 \%(1.9$ to 99$) 77 \%$ (72 to 81$) \quad 3.2(1.8$ to 4.2$) \quad 0.15$ (0.06 to 0.41 ) . High ed in Glossary; sensitivity, specificity, and Cl calculated from data in article: *Abbreviations defined in Glossary; sensitivity, specincity, and Cr calch

aninations of the legs for deep venous thrombosis permit anticoagulants to be withheld safely in this situation (2), but these tests are expensive and burdensome. It is in this context that Ginsberg and colleagues stadied a tapid whole-blood assay for b-dimer.

Their study provides new evidence that a negative test result for $\mathrm{D}$-dimer allows the physician to avoid further tests and anticongulation when the clinical pretest probability is low and the lung scan indicates a low or intermediate probability of acute $\mathrm{PE}$. Additional tests are necessary when the clinical probability is high, even if the $D-$ dimer result is negative and lung scans suggest a low probability of PE.

Practitioners must recognize that the bed- side D-dimer test is not a stand-alone screening test for acute $\mathrm{PE}$. They must also recognize that many D-dimer tests existand that most are insensitive screening tests for PE. Finally, practitioners should look for the results of additional clinical management studies, particularly in their ory or similar patient populations, before inctir porating the Dudimer assay into their algo rithm for the diagnosis of acute $\mathrm{PE}$. C. Gregory Elliots. Wh LDS Hospital and the University of Utal Salt Lake City, Utah, os

References

Hull RD, Raskob GE, Coates G, Panj AA. Chest. 1990;97:23-6.

2. Hull RD, Raskob GE, Ginsherg IS, et Arch Intern Med. 1994;154:289-97.

Evidence-Based Medicine of $84.8 \%$ and a specificity of $68.4 \%$. The sensitivity, specificity, and
lihood ratios for patients with low, moderate, and high pretest probabili 